\title{
Real-time Reliability Evaluation Based on Stochastic Degradation Process and Sequential Joint Distribution Estimation
}

\author{
QingZhen Gao ${ }^{1, a}$, LiHu Teng ${ }^{2}$, Zhuo Jiang ${ }^{3}$ and Hua Zhang ${ }^{4}$ \\ ${ }^{1}$ Anhui Polytechnic University, School of Mechanical and Automotive Engineering, $241000 \mathrm{Wu} \mathrm{Hu,} \mathrm{China}$ \\ ${ }^{2}$ Cheng Du Bureau of The Air Force, The Second Military Delegate Room, 550000 Gui Yang, China \\ ${ }^{3}$ Air Force Armament Department, Audit Division, 100000 Bei Jing, China \\ ${ }^{4}$ AVIC Guizhou Liyang Aviation Power Co., Ltd, 550000 Gui Yang, China
}

\begin{abstract}
According to the different performance degradation paths, reliability curves and parameters of each product in service phase, the reliability and remaining life of components at current moment can be evaluated in real-time reliability assessment by integrating real-time status, historical information and service time of components. The realtime reliability evaluation is valued because it is more personalized, precise, real-time and lean than traditional methods. Furthermore, the results from real-time reliability assessment can also represent the health status of component at current time. In the current research, real-time reliability assessment mainly relies on regression analysis and time series analysis, but these two methods are mainly used to describe the component degradation process, and cannot reflect the influence of external random environment on the component state change. At the same time, due to these limitations of time, economy and test conditions, it is also worth studying how to obtain more accurate and practical reliability distribution and determine a detection interval under the condition of less data. Therefore, based on the analysis of real-time reliability evaluation principle, a more appropriate real-time reliability evaluation method and the detection interval decision model are proposed by means of random degradation process, parameter sequence test joint distribution estimation and failure risk. The result from this method is a quantized value, which is convenient for the direct application of the follow-up maintenance decision research. Therefore, the research in this paper has extensive reference value and practical application prospect.
\end{abstract}

\section{Introduction}

Real-time reliability assessment ${ }^{[1]}$ refers to the real-time evaluation for reliability index based on real-time detection information from a specific component or system. The principle of real-time reliability evaluation is shown in Figure 1.

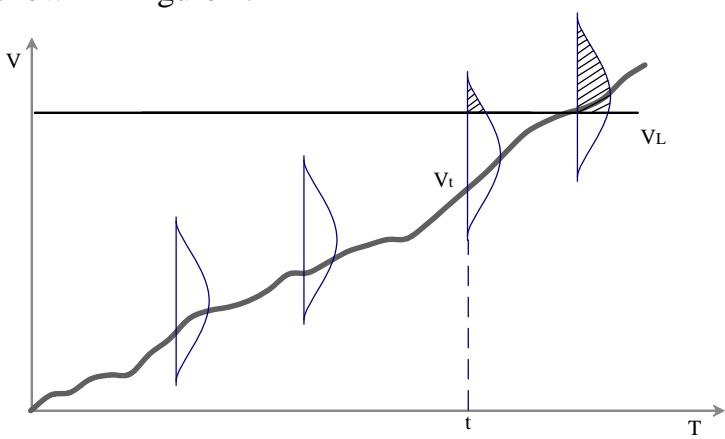

Figure 1. Schematic diagram of real-time reliability assessment of actual performance degradation process.

At any time $t$, the value of performance variable $V_{t}$ meets a certain distribution. In figure 1 , the value of hypothetical energy variable in the figure is normal distribution. And the fixed failure threshold $V_{L}$ is used as the failure distribution $F\left(t, V_{L}\right)=P\left(v(t)>V_{L}\right)$. That is the shaded part of the figure, so the real-time reliability is $R(t)=1-F\left(t, V_{L}\right)$.

Suppose that there is a kind of product after the experiment of large sample size, the performance degradation process and its distribution obtained by statistical analysis are shown in Figure 2.

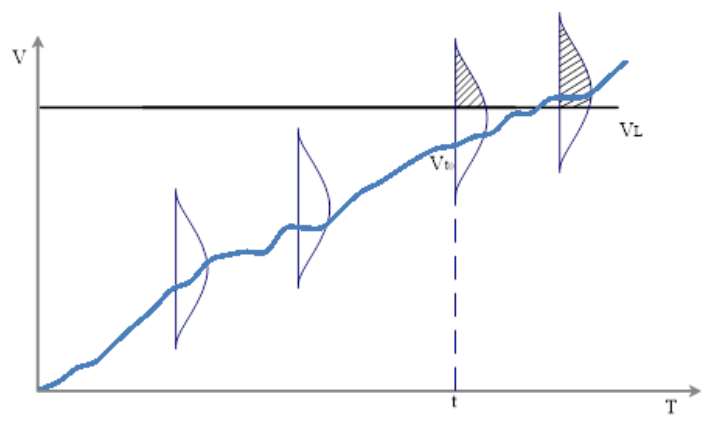

Figure 2. Performance degradation process and its prior distribution based on statistical analysis.

\footnotetext{
a Corresponding author: gaoqingzhen68@tom.com
} 
The shaded part in figure 1 can be considered as the average reliability of similar products at that time, and the average reliability is the result of statistical analysis. The performance degradation process of a real product in this category is showed in figure 1. Comparing figure 1 and figure 2, after the same time $t$, the statistical average degradation quantity is $V_{t_{0}}$, while the actual degradation quantity is $V_{t}, V_{t} \neq V_{t_{0}}$. Therefore, the distribution of performance parameters at this time in figure 1 should be different from that in figure 2. Comparing figure 1 and figure 2, since the actual degradation amount of the product is smaller than the average degradation amount. So the shaded part in figure 1 is smaller than the shaded part in figure 2. Which means the failure probability is small, that is the reliability is higher than the average reliability. The distribution of performance parameters in figure 1 includes both historical experimental data analysis and field measurement data. So it can better represent the actual situation of the product. However, the distribution of figure 1 is unknown.

The primary task of real-time reliability assessment is to obtain the prior distribution in figure 2 by statistical analysis of the historical information of similar products, and then to obtain the distribution of performance parameters in figure 1 which integrates real data with the state data measured in the field, so as to obtain the product real-time reliability.

The research methods of real-time reliability assessment mainly include performance degradation analysis method and real-time reliability index method. Among them, the performance degradation analysis method believes that the reliability decline of components or systems is caused by internal performance degradation. In other words, the product is considered to have failed when the performance degradation parameter value of a product reaches the pre-set threshold. Therefore, realtime reliability can be evaluated by establishing the corresponding performance degradation model.

Zhe Sun and linhai Zhao ${ }^{[2]}$ established an adaptive cloud model of failure efficiency between ambient data and component failure efficiency based on cloud theory. Zhixue Tan et al ${ }^{[3]}$ proposed a specific TLML algorithm to specified the loss function and alternative training scheme of component models for harnessing the information from sensor readings and empirical rules to serve the modeling. In literature ${ }^{[4]}$, an adaptive Krigingbased reliability analysis method was proposed to estimate the prior failure probability and the conditional probability about information. Its main contribution was a decomposition of rare joint failure event into two events both with relatively high probabilities. Zhiliang et $\mathrm{a}^{[5]}$ established a reliability model of marine power station under different working conditions and a reliability model database. Cai Baoping and Liu Yonghong ${ }^{[6]}$ proposed a novel real-time reliability evaluation methodology by combining root cause diagnosis phase based on Bayesian networks (BNs) and reliability evaluation phase based on dynamic BNs (DBNs). Yu Hongping and Tang $\mathrm{Mao}^{[7]}$ studied reliability assessment of multi-component systems under competing degradation and random shocks. Jinbo Huang et al ${ }^{[8]}$ presented a degradation model with a random failure threshold in reliability assessment by Bayesian approach.

In summary, regression analysis and time series analysis are mainly used to describe the degradation process for a system or component in current real-time reliability studies. However, these methods cannot reflect the influence of external random environment on component state change. Therefore, the methods of realtime reliability evaluation and detection interval decision are proposed in this paper based on random process, sequential joint distribution and failure risk theory.

\section{The Analysis of evaluation methods}

According to the principle of real-time reliability assessment, the complete reliability assessment process includes that firstly the appropriate degradation process is selected to represent the actual degradation process of components, then the prior distribution of unknown parameters in gradation process is determined and the posterior distribution is obtained by bayesian method, finally the reliability assessment model is derived based on failure threshold value. The specific steps are as follows:

1.The appropriate distribution $G(x, \theta(t))$ is selected to represent the distribution of performance degradation quantity of component at time, where $\theta(t)$ is the unknown random parameter, and the probability density function $G(x, \theta(t))$ is $g(x, \theta(t))$.

2.Based on the historical information of similar components, the prior density function $\pi\left(\theta\left(t_{i}\right)\right)$ of $\theta(t)$ at each time point $t_{i}$ is determined by statistical analysis.

3. According to the performance degradation quantity $V_{i}$ actually measured at time $t_{i}$ of the component, the unknown parameter posterior density function $\pi\left(\theta\left(t_{i}\right) \mid V_{i}\right)$ is obtained by bayesian method, then the probability density function $g\left(x, \theta\left(t_{i}\right) \mid V_{i}\right)$ of the performance degradation quantity is obtained.

4. According to $g\left(x, \theta\left(t_{i}\right) \mid V_{i}\right)$ and the set failure threshold $l$, the component reliability $R\left(t_{i}\right)$ at time $t_{i}$ is obtained, that is the real-time reliability evaluation model for the component at time $t_{i}$.

\subsection{The model of stochastic degradation process}

The component performance degradation and failure is a slow development process. Environmental stress causes component to produce wear and damage continually. Wear and damage accumulation process is the component degradation process. A lot of components are subjected to continuous impact damage in actual work environment, such as vibration damage, overload damage, etc. The occurrence of impact damage may be time continuous or discrete random. When the cumulative damage reaches a certain level, it leads to component failure.

For the stochastic process model of continuous damage accumulation, Wiener-einstein degradation 
failure model and Gamma degradation failure model ${ }^{[9]}$ are the main models. Since most actual degradation processes are monotonous and random, so their increments are nonnegative and independent. It is very appropriate to use Gamma processes to describe their degradation processes.

It is assumed that the performance parameter variable $X(t)$ obeys Gamma distribution at any time $\mathrm{t}$ and its distribution density function is:

$f_{X(t)}(x)=G a(x \mid v(t), u)=\frac{u^{v(t)} x^{v(t)-1} \mathrm{e}^{-u x}}{\Gamma(v(t))} I_{(0, \infty)}(x)$

Where, $I(\cdot)$ is the function which is constant 1 but not constant 0 in the domain, $\Gamma(\cdot)$ is the Gamma function, $v(t)$ and $u$ are the shape parameter and scale parameter of the Gamma distribution function $\mathrm{Ga}(\cdot)$ respectively, $v(t)$ is the continuous and non-subtractive real function, $v(0) \equiv 0$, and $u$ is the real number greater than zero. Assuming that the Gamma process is a stationary process and it can be set $v(t)$ as a linear function $v(t)=a t$, where $a$ is a constant. The expectation of performance parameter variable $X(t)$ is:

$$
E(X(t))=\int_{0}^{\infty} x f_{X(t)}(x) d x=\frac{v(t)}{u}=\frac{a t}{u}
$$

The variance is:

$$
E\left((X(t)-E(X(t)))^{2}\right)=\frac{a t}{u^{2}}
$$

Let $E(X(t))=s t, E\left((X(t)-E(X(t)))^{2}\right)=m^{2} t$, and $m=v s, v$ is a constant, then the $X(t)$ distribution function expression (3) can be transformed into $f_{X(t)}(x)=G a\left(x \mid t / v^{2}, 1 / s v^{2}\right)$. At this point, the unknown random parameter of distribution function is $s$.

\subsection{The sequential distribution estimation}

For a specific function, the form of prior distribution can be obtained by the conjugate distribution method, and the inference process from prior distribution to posterior distribution can be simplified. Firstly, the conjugate prior distribution form of parameter $s$ is assumed, and then the parameter is determined according to historical information, that is the prior distribution of parameter $s$.

Let the prior distribution of parameter $s$ be the inverse Gamma distribution, and the probability density function is:

$$
\pi(s)=\operatorname{Ig}(s \mid \delta, \eta)=\frac{\eta^{\delta}}{\Gamma(\delta)}\left(\frac{1}{s}\right)^{\delta+1} \mathrm{e}^{-\frac{\eta}{s}} I_{(0, \infty)}(x)
$$

Where: $\delta$ and $\eta$ are constant respectively, the general determination method is to use historical information to fit.

Assuming the actual quantity of degradation is detected at time $t$ is $y$, then the conjugate posterior probability density function of $s$ is:

$$
\begin{aligned}
& \pi(s \mid y)=\frac{f(y \mid s) \pi(s)}{\int_{0}^{+\infty} f(y \mid s) \pi(s) d s} \\
& =\frac{\left(\frac{y}{v^{2}}+\eta\right)^{\left(\frac{t}{v^{2}}+\delta\right)}}{\Gamma\left(\frac{t}{v^{2}}+\delta\right)}\left(\frac{1}{s}\right)^{\frac{t}{v^{2}}+\delta+1} \mathrm{e}^{-\frac{y}{v^{2}}+\eta} \frac{I_{(0, \infty)}}{s}(x) \\
& =\operatorname{Ig}\left(s \mid \frac{t}{v^{2}}+\delta, \frac{y}{v^{2}}+\eta\right)
\end{aligned}
$$

It is assumed that there are $j$ actual test data $y_{1}, \mathrm{~L}, y_{j}$ at the existing time $t_{1}, \mathrm{~L}, t_{j}$, and the posterior joint distribution of parameter $s$ is:

$$
\begin{aligned}
& \pi\left(s \mid y_{1}, \mathrm{~L}, y_{j}\right)=\operatorname{Ig}\left(s \mid \delta+\sum_{i=1}^{j} \frac{t_{i}-t_{i-1}}{v^{2}}, \eta+\sum_{i=1}^{j} \frac{y_{i}-y_{i-1}}{v^{2}}\right) \\
& =\operatorname{Ig}\left(s \mid \delta+\frac{t_{j}}{v^{2}}, \eta+\frac{y_{j}}{v^{2}}\right)
\end{aligned}
$$

The above formula indicates that the calculation of posterior probability density function requires only the last detection result, and the estimated value of posterior expectation for $s$ is:

$$
\hat{s}=\frac{\eta+y_{j} / v^{2}}{\delta+t_{j} / v^{2}-1}
$$

The formula (7) is substituted into equation (3) to obtain the posterior distribution density function of performance parameter variables.

$$
f_{X(t)}(x)=\frac{\left(1 / \hat{s} v^{2}\right)^{t / v^{2}} x^{t / v^{2}-1} \mathrm{e}^{-x / \hat{s} v^{2}}}{\Gamma\left(t / v^{2}\right)} I_{(0, \infty)}(x)
$$

\subsection{The real-time reliability assessment}

Suppose the degradation failure threshold of component is $l$, then the detection result $y_{j}$ at $t_{j}$ moment can be calculated according to the reliability definition.

$$
\begin{gathered}
R\left(t_{j}\right)=P\left\{x\left(t_{j}\right)<l\right\}=\int_{0}^{l} f_{X\left(t_{j}\right)}(x) d x \\
=\int_{0}^{l} \frac{\left(1 / \hat{s} v^{2}\right)^{t_{j} / v^{2}} x^{t_{j} / v^{2}-1} \mathrm{e}^{-x / \hat{s} v^{2}}}{\Gamma\left(t_{j} / v^{2}\right)} d x
\end{gathered}
$$

The formula (9) is the real-time reliability evaluation model for component with the detection result $y_{j}$ at time $t_{j}$.

\subsection{Determination of detection interval based on failure risk}

Based on the above real-time reliability research, the failure conditional probability is $F(t+\Delta t \mid t)$ in the next time period $\Delta t$. If the component is detected and found to be working normally from time $t$. Suppose the life of 
component is $T$, then according to conditional probability, the risk of component failure can be expressed as:

$$
F(t+\Delta t \mid t)=\frac{P(t<T)-P((t+\Delta t)<T)}{P(t<T)}
$$

According to the reliability definition $P(t<T)=R(t)$, formula (10) can be evolved into formula (11).

$$
F(t+\Delta t \mid t)=\frac{R(t)-R(t+\Delta t)}{R(t)}
$$

Assume the fault risk threshold is constant $D$, that is the fault risk is not greater than $D$. In general, as long as an appropriate $\Delta t$ is found, so that the fault risk within the range of $\Delta t$ is equal to the fault risk threshold $D$. Then this $\Delta t$ is the optimal detection interval. The $\Delta t$ meets the requirement of the longest detection interval under fault risk constraint, then formula (11) is converted to formula (12).

$$
\frac{R(t)-R(t+\Delta t)}{R(t)}=D
$$

Since the increment of Gamma process is independent and non-negative, it is very suitable for describing the random process of monotone degradation. Hypothesis: (1) At any measurement time $t$, the performance parameter variable $X(t)$ follows the Gamma distribution. (2) The traditional reliability function $R_{c}(t)$ of component obeys Weibull distribution, that is $R_{c}(t)=\exp \left(-(t / \lambda)^{\beta}\right)$.

Therefore, the first detection interval $t_{1}$ of the component is:

$$
t_{1}=\lambda(-\ln (1-D))^{\frac{1}{\beta}}
$$

According to the real-time reliability function $R(t)=\exp \left(-(t / \hat{\lambda})^{\hat{\beta}}\right)$, the detection interval $\Delta t_{i}$ determined at time $t_{i}$ after the first state detection is :

$$
\Delta t_{i}=\hat{\lambda}\left(\left(\frac{t_{i}}{\hat{\lambda}}\right)^{\hat{\beta}}-\ln (1-D)\right)^{\frac{1}{\hat{\beta}}}-t_{i}
$$

\section{An example analysis}

The table 1 is the data record of percentage change with time about working current at the temperature of $90^{\circ} \mathrm{C}$. The working current data is from an engine pressure sensor that is a high reliability and long life component. A total of 15 samples were tested, and the data were tested every 500 hours until 4000 hours. It is assumed that the failure threshold of the sensor is 10 .

Table 1. A record of percentage change with time about working current of a sensor at $90^{\circ} \mathrm{C}$.

\begin{tabular}{|c|c|c|c|c|c|c|c|c|c|}
\hline & 0 & 500 & 1000 & 1500 & 2000 & 2500 & 3000 & 3500 & 4000 \\
\hline 1 & 0 & 0.95 & 2.75 & 4.36 & 5.50 & 6.75 & 8.03 & 9.52 & 10.97 \\
\hline 2 & 0 & 1.24 & 2.33 & 3.77 & 5.02 & 6.09 & 7.19 & 8.45 & 9.31 \\
\hline 3 & 0 & 1.19 & 2.01 & 2.99 & 3.96 & 4.47 & 5.29 & 6.03 & 6.91 \\
\hline
\end{tabular}

\begin{tabular}{|c|c|c|c|c|c|c|c|c|c|}
\hline 4 & 0 & 0.64 & 1.97 & 2.97 & 3.82 & 4.53 & 4.50 & 5.64 & 6.17 \\
\hline 5 & 0 & 0.63 & 1.77 & 2.60 & 3.40 & 4.66 & 5.64 & 6.35 & 7.62 \\
\hline 6 & 0 & 1.41 & 2.91 & 3.84 & 5.37 & 6.73 & 8.65 & 9.99 & 11.04 \\
\hline 7 & 0 & 0.95 & 1.49 & 2.42 & 2.97 & 4.51 & 4.87 & 5.58 & 7.19 \\
\hline 8 & 0 & 1.09 & 1.79 & 2.43 & 3.05 & 3.77 & 4.78 & 5.48 & 6.27 \\
\hline 9 & 0 & 0.95 & 1.98 & 3.45 & 4.14 & 4.94 & 5.87 & 6.88 & 7.81 \\
\hline 10 & 0 & 1.51 & 3.03 & 4.52 & 6.28 & 7.82 & 8.95 & 10.47 & 12.25 \\
\hline 11 & 0 & 1.02 & 1.99 & 2.86 & 4.04 & 4.83 & 5.68 & 6.56 & 7.46 \\
\hline 12 & 0 & 0.82 & 1.76 & 3.63 & 4.46 & 5.25 & 5.99 & 7.02 & 7.91 \\
\hline 13 & 0 & 0.76 & 1.87 & 2.97 & 3.95 & 5.50 & 6.54 & 7.42 & 8.93 \\
\hline 14 & 0 & 0.72 & 1.37 & 2.58 & 3.42 & 4.51 & 5.44 & 6.17 & 6.70 \\
\hline 15 & 0 & 0.85 & 1.56 & 2.30 & 3.46 & 4.13 & 4.65 & 5.88 & 6.66 \\
\hline
\end{tabular}

From the 135 data in table 1 , the trait of performance data degradation with time can be seen: The initial quantities are all 0; Monotonic growth; Increments are independent. Thus the degradation of sensor performance can be described by the Gamma process.

After the Gamma process model was established, the prior distribution of parameter $s$ must be determined. According to equation (4), the prior distribution $s$ is determined by $\delta$ and $\eta . \delta$ and $\eta$ can be estimated from the data in table 1 . After analysis, the $\delta=1.4, \eta=6$ are taken for inverse Gamma distribution as the prior distribution of parameter $s$. The reliability evaluation model for working current of the sensor at $90^{\circ} \mathrm{C}$ can be obtained in formula (15) from equations (7) and (9) by taking $v=1$.

$$
R\left(t_{j}\right)==\int_{0}^{l} \frac{\left(\frac{1.4+t_{j}-1}{6+y_{j}}\right)^{t_{j}} x^{t_{j}-1} \mathrm{e}^{-\frac{1.4+t_{j}-1}{6+y_{j}}}}{\Gamma\left(t_{j}\right)} d x
$$

It can be seen that the reliability assessment model not only considers the time factor, but also considers the real-time test data.

The data at 2000 hours from the fifth sample is used for experimental calculation. By substituting $l=10$, $t_{200}=2000, y_{2000}=3.40$ into the equation, the real-time reliability at that time $R\left(t_{2000}\right)=0.9980$ can be obtained.

The traditional reliability assessment ${ }^{[10]}$ was finished based on the data in table 1. At a given time $t$, the traditional reliability is $R^{\prime}(t)=\exp \left(-(t / 5818.6)^{5.62}\right)$. If the date at 2000 hours is substituted in this equation, the average reliability of these 15 samples at 2000 hours is $R^{\prime}(2000)=0.9974$.

Comparing the real-time reliability of the fifth sample with the average reliability from all 15 samples, it can be easily seen that the degradation rate of the fifth sample is slower than the average speed. So the real-time reliability of the fifth sample is higher than the average reliability at 2000 hours. In other words, the fifth sample was healthier than average at 2000 hours.

Assuming that the traditional reliability curve of the sensor follows weibull distribution, where $\lambda=5676.5$, $\beta=5.85$. Then seven data points are taken from it to form an initial data sequence. $E_{0}=\{(1000,0.9999)$, (1500,0.9995), (2000,0.9975), (2500,0.9913), $(3000,0.9761), \quad(3500,0.9441),(4000,0.8854)\}$. Suppose 
that the failure risk constraint $D=0.1$ during the detection interval. The $\lambda, \beta$ and $D$ are substituted into equation

(13) to obtain the first detection interval $t_{1}$ (Per unit time).

$$
t_{1}=5676.5(-\ln (1-0.1))^{\frac{1}{5.85}} \approx 3800
$$

By taking $v=1$, the real-time reliability $R\left(t_{1}\right)=0.9565$ of the sensor in the first state detection can be obtained from formula (9). And $\hat{\beta}=7.6572$, $\hat{r}=65.5489, \hat{\lambda}=5500$ can be calculated from $E_{0}$ and the real-time reliability function $R(t)=\exp \left(-(t / \hat{\lambda})^{\hat{\beta}}\right)$. The $\hat{\beta}, \hat{r}, \hat{\lambda}$ and $t_{1}$ are substituted into equation (14) to obtain detection interval $\Delta t_{1}=550.0$ (Per unit time) after the first state detection.

\section{Conclusion}

Based on studying the principle of real-time reliability assessment, a more suitable method is proposed in this paper for real-time reliability assessment and detection interval prediction of components by integrating real-time status, historical information, environmental factors and service time of components.

In this method, stochastic process is used to describe the component degradation process to make it more realistic. The problem that a component reliability distribution is difficult to obtain under the condition of less data is solved by successive joint distribution estimation. The calculation methods for the first and subsequent component detection interval are established by introducing the concept of failure risk. The above research provides reference for evaluating a component reliability in real time and scientifically.

The working data of a sensor was taken as the research object. Then according to the real-time reliability evaluation method in this paper, the real-time health status of the sample was obtained and the first and subsequent detection interval were calculated. These works verified the correctness and effectiveness of the research.

The results from this real-time reliability evaluation method and detection interval decision model are quantized values, which is convenient for the direct application of the follow-up maintenance decision research. At the same time, these factors such as the availability, accessibility and cost of information collection are fully taken into account in the method, so that the method has a good practical value and application basis.

\section{References}

1. Chunhui Liang. The research on real-time reliability analysis and prediction to spindle bearing system of wind turbine $[J]$. Changchun Inst. Tech. (Nat. Sci. Edi.), 2017, Vol.18, No.3: 41-45.

2. Zhe Sun, linhai Zhao. Real-time evaluation method of JTC reliability based on cloud theory[J]. Railway journal, 2019, 18:53-73.

3. Zhixue Tan, Shisheng Zhong, Lin Lin. Trans-layer model learning: A hierarchical modeling strategy for real-time reliability evaluation of complex systems [J]. Engineering and System Safety, 2019, 2(4) : 382-393.

4. Zeyu Wang, Abdollah Shafieezadeh. Real-time highfidelity reliability updating with equality information using adaptive Kriging $[\mathrm{J}]$. Reliability Engineering and System Safety, 2020, 3 (1) :154-163.

5. Zhiliang, WuYubin, YaoDan Wang. Design and Study of Real-time Online Assessing System for Marine Power Station Reliability [J]. 2017 2nd International Conference on Automation, Mechanical and Electrical Engineering, 2017: 165-168.

6. Cai Baoping, Liu Yonghong. Real-time reliability evaluation methodology based on dynamic Bayesian networks: A case study of a subsea pipe ram BOP system. [J]. ISA transactions, 2015, 5 (4): 353-357.

7. Yu Hongping, Tang Mao. Reliability assessment for systems suffering competing degradation and random shocks under fuzzy environment[J]. Science progress, 2019,10(1):166-179.

8. Jinbo Huang, Dejing Kong, Lirong Cui. Bayesian reliability assessment and degradation modeling with calibrations and random failure threshold[J]. Journal of Shanghai Jiaotong University (Science), 2016, Vol.21 (4), pp.478-483.

9. Wang Xiaolin, Guo Bo, Cheng Zhi. Real-time reliability evaluation for product with nonlinear driftbased Wiener process[J]. Journal of Central South University (Science and Technology), 2013(8), Vol.44, No.8:3203-3209.

10. Wang Haoming, Tang Chong, Wu Liping, Yang Lei, Yao Ying, LiuHong. Dynamic Reliability Assessment of Distribution Network Based on Equipment State Evaluation Model[J]. Proceedings of the CSU-EPSA, 2017 (7), Vol.29, No.7: 68-74.

Project support: 2019 talent fund project of Anhui Polytechnic University (2019YQQ001) 